\title{
Ubiquitous Computing, Customer Tracking, and Price Discrimination
}

\author{
Alessandro Acquisti \\ H. John Heinz III School of Public Policy and Management \\ Carnegie Mellon University*
}

Revised: January 24, 2005

To appear in: G. Roussos (ed), Ubiquitous Commerce, Springer Verlag, 2005

\section{Introduction}

The availability and cost-efficiency of modern information and communication technology have made "interactive marketing" and individual customer addressability not only possible but economical. In 1991, Blattberg and Deighton Blattberg and Deighton 1991] defined the new frontiers for marketing opened by interactive computer technologies as the "age of addressability." Today, ubiquitous computer systems make it possible for consumers and providers of services and goods to engage in repeated, seamless interactions regardless of their respective physical locations.

Ubiquitous computing refers to methods of enhancing computer use by making networks of sensors and computers available and embedded in the physical environment Weiser 1993. The technologies on which ubiquitous computing applications are based span automatic identification (Auto-ID), such as Radio Frequency Identification (RFID); (wireless) communication systems, such as Global Standard for Mobile Communication (GSM); positioning services, such as Global Positioning System (GPS); and sensor networks. Together, these technologies are making new or improved business models, services, and products possible, and attention in the academic literature is naturally growing towards the business opportunities of ubiquitous computing. The volume of ubiquitous commerce, in particular ("any transaction with a monetary value that is conducted using ubiquitous computing technology" Roussos 2004) is expected to increase significantly in the coming years as e-commerce and wireless technologies continue to expand (the m-commerce or mobile commerce market alone is expected to be worth over $\$ 50$ bn by 2009 Carr 2004]). Ubiquitous commerce involves transactions as diverse as mobile phone based purchases, "intelligent"

\footnotetext{
${ }^{*}$ Email contact: acquisti@andrew. cmu.edu.
} 
shopping carts, context-aware wallets, and other applications that have only started being explored. In commerce scenarios, ubiquitous computing devices will act as channels for sellers' services, as sensors of environmental and customers' conditions, and as "effectors" mediating between the customer's needs and the provider's services (see also Gershman [2002]).

Advancements in information and communication technologies give companies better tools to study their customers, perfect their marketing strategies, and dynamically change their value propositions based on the information collected. In ubiquitous computing environments, customers may be uniquely identified and recognized by ubiquitous computing sensors because of the devices they are carrying. Based on the analysis of individual data, sellers in industries adopting these technologies may offer to each individual a different service - depending on factors such as previous purchase history, location, or other personal or environmental traits. Customers may receive "on the spot" personalized promotions, discounts, as well as targeted products, differentiated content, and individualized information - on their own mobile computing devices, based on the information they are revealing through their presence in a network of sensors.

These scenarios open new revenue opportunities for technologically savvy sellers but also raise new trade-offs for buyers.

On the seller's side, of particular interest to this chapter's analysis is the possibility of combining context, historical, location and other personal data to dynamically alter the price of a product for each consumer - a form of price discrimination also known as dynamic pricing. On the Internet there have been accounts of attempts at dynamic pricing in the past (see Streifield [2001] and Taylor 2002 ). Pervasive computing environments, because of their ubiquitousness and invisibility, offer sellers new powerful tools to quietly implement such pricing strategies.

On the consumer's side, ubiquitous computing technologies offer promises and opportunities and also some risks - notably, privacy invasions and, in fact, price discrimination Acquisti 2002]. Nobody likes to pay for the same product more than the amount the other person spent. Faced by intrusive information policies and price discriminating strategies, however, consumers can decide to bypass the seller's tracking attempts through privacy enhancing and anonymizing technologies, or to avoid the seller altogether.

In prior work on intertemporal price discrimination Acquisti and Varian [2005], we addressed some of the pricing issues associated with generic tracking technologies. We used models of repeated interactions between sellers and their customers in which sellers use various information technologies to track customers over time (such as Internet "cookies" in online shopping), and customers use strategies (such as delaying purchases, selecting a different vendor, or adopting anonymizing or privacy technologies) to avoid being tracked. The models highlighted the conditions under which sellers find it optimal to use tracking data about their customers for price discrimination, and the conditions under which customers find it optimal to reveal or hide their identities.

In this chapter we apply and extend that analysis to ubiquitous computing 
scenarios, in which sellers may use customer personal, history, or context data for price discrimination. To this goal, we first discuss the features of ubiquitous computing technologies of interest to commerce (Section 22). We then focus on the application of ubiquitous computing technologies for customer tracking and price discrimination, and we investigate the consequences for buyers and sellers of the application of these technologies. We first summarize the single good model presented in Acquisti and Varian 2005 for the dynamic pricing case, and we show how it can be applied to ubiquitous computing environments (Section 3). We then extend the model to consider various cost specifications, multiple goods, and additional marketing strategies that may be natural applications in ubiquitous computing environments (Section 4). Finally, we summarize the implications of our model in the context of ubiquitous computing commerce (Section 5).

In addition to the literature on ubiquitous computing commerce that we discuss in the next sections, our analysis naturally touches different areas of the economic and marketing literature: the literature on intertemporal price discrimination (in particular Stokey 1979, Salant 1989, Riley and Zeckhauser [1983], Hart and Tirole 1988, , Villas-Boas [1999], and Villas-Boas |2004]); the literature on consumer addressability (in particular Blattberg and Deighton [1991], McCulloch et al. [1996], Rossi and Allenby [1999], Ulph and Vulkan 2000, 2001, , Chen et al. [2001], and Chen and Iyer [2002]); and the literature on economic aspects of personal privacy (in particular Calzolari and Pavan 2001] and Taylor 2002]). In what follows we will only discuss related articles when relevant to the model. For a more complete review of the literature we refer the reader to Acquisti and Varian 2005.

\section{Ubiquitous Computing and Commerce}

Ubiquitous computing applications rely on networks of computing, sensing, and communicating devices that are often mobile, wireless, and embedded in the environment. Since Mark Weiser's Weiser 1993 seminal work, research on those applications has steadily increased, but only recently the literature has focused on their commercial and business aspects: see Fleisch 2001, Gershman [2002], Kourouthanassis and Roussos [2003a], Christ et al. [2003], [Fleisch and Tellkamp [2003], and Kourouthanassis and Roussos [2003b].

According to Christ, Fleisch, and Mattern Christ et al. 2003], ubiquitous computing "is the basic technology behind the next innovation thrust to follow eBusiness." The combination of automatic identification technologies, (wireless) communication technologies, positioning services, and sensor network technologies should lead to new business processes in areas as diverse as supply chain management, customer relationship management, and retailing.

Christ, Fleisch, and Mattern Christ et al. 2003 believe that sensor networks may recognize or cause modifications in environmental conditions and allow decision making based on real time environmental data, leading to cost savings and quality improvements. Ubiquitous computing may integrate businesses' in- 
formation systems with the actual operational environment of the firm, thereby closing the "gap between information system and reality" Christ et al. 2003 . One significant contribution of ubiquitous computing technologies may be the reduction of the marginal cost of the integration of the real and the virtual world, enabling "information systems to collect more detailed data at the point of creation (POC) and eventually [allowing] managers and machines at the point of action (POA) to implement decisions based on high quality, real-time information" (Fleisch and Tellkamp forthcoming, 2005). These improvements may lead to better information systems integration, data quality improvements, and digital management control loops.

In addition to optimization of business processes, other researchers believe that ubiquitous computing may make new sources of revenue possible through novel services and enhanced products. Of particular interest here are the consequences of ubiquitous technologies on commerce and retailing. Gershman 2002 noted that commerce will be affected by the ability of ubiquitous computing technologies to provide service channels from the provider to the user, to inform users about those services, and to act as "effectors" of the user's needs to affect the environment. For example, Christ, Fleisch, and Mattern Christ et al. 2003 note that ubiquitous computing could be used to track inventory and decrease the costs associated with out of stock or dead stock material, as well as to increase customer confidence in the quality of a product and trust in the seller. Kourouthanassis and Roussos Kourouthanassis and Roussos 2003a propose that context aware ubiquitous computing applications could provide shoppers personalized services, promotional information, and offers based on the recognition and tracking of their identities, current choices, and other characteristics (such as location). For example, Gillette's s collaboration with Wal-Mart and Tesco focuses on "smart shelves" applications that increase availability of Gillette products and attempt to reduce theft Christ et al. 2003. Prada New York store uses RFID tags on clothing items to display information about them on a screen Christ et al. 2003. MyGrocer, an ubiquitous computing application for the grocery sector, focuses on home replenishment schema and service quality enhancement in smart-home and in-store scenarios Kourouthanassis and Roussos 2003ab. In the in-store scenarios, "RF tags uniquely identify each product and are constantly transmitting the presence of the product to RF-receivers, effectively positioned on the shopping cart. When the consumer enters the supermarket she logs in MyGrocer through her cart. The system identifies the user and displays her shopping list (missing products) to the shopping carts display screen"Kourouthanassis and Roussos 2003a. This project found Kourouthanassis and Roussos $[2003 \mathrm{~b}$. that such shopping features made possible by ubiquitous computing technologies "provide a more entertaining and stress-free shopping trip compared to conventional shopping, and thus transform a utilitarian activity into an opportunity for entertainment."

However Fleisch and Tellkamp forthcoming, 2005] warn that identifying the value-creating applications is not trivial. They suggest, for example, changing a company's business model from selling services at a fixed price to usage-based billing. But aggressive pricing strategies may end up alienating customers. In 
this chapter we study the incentives for sellers to use ubiquitous computing tracking technologies to enforce price discrimination, and the possible reactions by their customers. Thanks to modern information and communication technologies, companies can gather massive amounts of data about consumers, infer from that data trends in consumer behavior, and then attempt to use their analysis to increase their profits. This may certainly involve the ability to discern the customer's reservation price for a certain good, and thereafter the ability to dynamically change prices of the same product for each individual customer based on that information. However, standard micro-economics analysis can be used to show that these forms of price discrimination per se are not optimal: a seller facing strategic customers (whose taste it does not know in advance) cannot do better than committing to optimal single period contracts (see for example Hart and Tirole 1988, and below). Why and how exactly should sellers use ubiquitous computing technologies to track customer information and use it dynamically to maximize their profits, without alienating buyers with aggressive price discriminative policies?

In the rest of this chapter we use tools from micro economic theory to examine the conditions under which sellers will find it optimal to engage in price discrimination when consumers can adopt strategies to protect their privacy, and whether a balance can be found between sellers' and buyers' different needs.

\section{Tracking and Price Discrimination}

By tracking and analyzing individual consumer data, sellers can implement interactive marketing strategies and present to each individual a personalized offer. In particular, sellers can use consumer information (combining context, historical, location and personal data) for price discrimination.

An intriguing result in the micro economic literature, however, shows that price discrimination is not an optimal strategy when the seller can commit to prices and consumers have stationary valuations for the good: intertemporal price discrimination cannot do better than fixed prices strategies (see Stokey [1979], Salant 1989 and Riley and Zeckhauser 1983]).

Acquisti and Varian Acquisti and Varian 2005 show that the same result applies when customers can be individually tracked through information technology. They use a simple model of dynamic pricing that can be applied to the ubiquitous computing tracking scenario, with a single profit-maximizing seller of a good that can be provided at zero marginal cost. The seller has some mechanism for recognizing, tracking, and recording purchase histories of customers (in the ubiquitous computing case, for example, the mechanism could be a network of RFID sensors and other mobile devices in a supermarket that detect and react to Auto-ID devices used or carried by the consumer and associated with her identity - for example, an RFID tag on her shopping cart). We can refer to this mechanism simply as an automatic "ID". Consumers may sometimes have mechanisms to avoid being tracked (for example, the RFID "killer" tag that disrupts the radio communication between the tag and the sensors). We will 
loosely refer to such mechanisms simply as "anonymizing technologies."

Imagine that customers are rational and forward looking (they know that their current behavior will affect the vendor's future choices). We limit our analysis to two shopping opportunities, or "visits." In other words, we assume that consumers visit the store (the ubiquitous computing commerce environment) at most twice. When they visit the seller for the first time, their personal information or ID is sensed and registered, and a price for the good of interest to the consumer is presented (for example on her mobile computing device or through other ubiquitous computing tools: Christ et al. 2003 observe: "[c]hanging the price labels is no longer necessary as all the shelves [may be] equipped with labels made from electronic paper which immediately show the modified price."). The consumer's decision about whether to purchase at that price is observed and recorded by the seller. The second time the consumer comes into the same ubiquitous computing environment for another purchase, the price she is offered can be conditioned on her earlier behavior (recorded by the system and associated to the consumer ID).

Imagine that there exist two consumer types - one type values the good $v_{H}$, more than the other type, $v_{L}$ - and that a fraction $\pi$ of the population has the higher willingness to pay for one unit of the good. The seller could set a flat price at each period (either pricing at the lower valuation, getting all customers; or at the higher valuation, getting only the high valuation customers). Or, the seller may change prices dynamically across the two shopping opportunities, "conditioning" them on the reaction of customers to the prices in the first period. Since high-value forward looking consumers will realize that purchasing at a high price may guarantee that they will face a high price in the future, they may adopt some anonymizing technology to avoid establishing a purchase history or they may just delay purchase (by refusing an offer at a certain price in the first purchasing experience).

For this model, Acquisti and Varian 2005. prove that there is one undominated discriminatory pricing policy for the seller, which leads to the high value consumer type purchasing in both periods, and the low valuation type purchasing only once. It also turns out, however, that the profit from the discriminatory policy never exceeds the profit from flat pricing 11 When selling to high-value customers is more profitable than selling to every consumer at the lower price, the seller will prefer to only sell to high-value consumers at the high price (rather than providing discounts to also attract the low valuation type). In other words, it appears that using tracking data for price discrimination may not lead to higher profits for the seller.

\section{Interactive Marketing and Optimal Profit}

If simple price discrimination based on customer tracking is not optimal with rational consumers, in what ways can merchants use tracking data and interactive marketing to enhance their profits? How can they optimally use personal

\footnotetext{
${ }^{1}$ See Acquisti and Varian 2005 for the mathematical details.
} 
information such as purchase history, ubiquitous computing context data, and revealed preferences?

\subsection{Making it costly to hide previous behavior}

First of all, the merchant may make it costly for the customer to hide previous behavior in a ubiquitous computing environment (for example her purchase history).

While sophisticated consumers may always delay purchases at little or no cost, they may face costs when trying to hide their purchase history through anonymizing technologies (for example, their previous high evaluation and purchase of a certain product). The merchant could structure the ubiquitous computing environment precisely in a way that it makes the adoption of privacy enhancing, anonymizing strategies or technologies costly (for example, by requiring customers in a supermarket to carry and pay only through personally identifiable wireless devices). The consumer may still bypass these efforts, but at a cost.

We can model the cost of using a generic form of anonymizing technology. Imagine that $f$ denotes the cost of a technology that allows the buyer to present herself as a "first time customer" in the model discussed in Section 3 even after repeated purchases. This means that when the seller sees a customer with "no ID," it cannot be sure whether that is really a new customer, or an old one who deleted her ID. Assume that if consumers have no ID information indicating a prior visit, they are charged a price $p_{0}$; if they have an ID indicating that they bought on a prior visit, they are charged $p_{b}$; if they have an ID indicating that they did not buy on their earlier visit, they are charged $p_{n}$.

The relevant parameter values for $f$ are therefore those where

$$
2\left(v_{H}-v_{L}\right)>f>0 .
$$

We are interested in studying the price discriminatory strategy for the seller in which the high-value type consumes both periods and the low-value type consumes only in the first period. This leads to a simple mechanism design problem Fudenberg and Tirole 1991] with the following incentive compatible and individual rationality constraints:

$$
\begin{aligned}
2 v_{H}-p_{0}-p_{b} & \geq 2 v_{H}-2 p_{0}-f \\
2 v_{H}-p_{0}-p_{b} & \geq 0 \\
v_{L}-p_{0} & \geq 2 v_{L}-2 p_{0}-f \\
v_{L}-p_{0} & \geq 0 .
\end{aligned}
$$

The first inequality says that the high-value consumer must prefer to purchase at $p_{0}$, have her ID information recorded, and pay price $p_{b}$, rather than remove the ID information and incur the cost $f$. The second inequality says that the high-value consumer must get non negative utility from buying twice 
at the given prices. The third and forth inequalities make similar statements for the low-value consumer.

The solution to these inequalities is

$$
\begin{aligned}
& p_{0}=v_{L} \\
& p_{b}=v_{L}+f,
\end{aligned}
$$

which leads to profit of $v_{L}+\pi\left(v_{L}+f\right)$. For example, the seller can charge $v_{L}$ the first time a customer comes around, and then $v_{l}+f$ the second time around. Note that this is almost the same as flat pricing at $v_{L}$, at least for small values of $f$.

When is this profit larger than the profit resulting from charging a flat price of $v_{L}$ ? One needs

$$
v_{L}+\pi\left(v_{L}+f\right)>2 v_{L},
$$

which reduces to

$$
f>\frac{1-\pi}{\pi} v_{L} .
$$

When is this profit larger than flat pricing at the high price? When

$$
v_{L}+\pi\left(v_{L}+f\right)>2 \pi v_{H},
$$

which reduces to

$$
f>2 v_{H}-\frac{1+\pi}{\pi} v_{L} .
$$

Equations (7) and (8) describe a set of relationships among the parameters that determine the optimality of the conditioning solution when there is a cost to avoid being identified by ubiquitous computing (or any other tracking) technologies. When the cost of concealing information (i.e., anonymizing) are high, price conditioning based on a "low price first, then high price" strategy can become profitable even with sophisticate consumers, who will just let sellers track them over repeated transactions rather than going through the hassle of using an anonymizing technology.

This is not surprising: if anonymizing technologies are deemed too expensive, consumers simply will not use them. But then, perhaps more interestingly, how expensive are these technologies?

The cost $f$ of an anonymizing technology is not only its monetary price, but also the inconvenience (such as usage costs, learning curves, and other hassles) that customers have to incur to use it. Even when small, these costs might be enough to turn customers away from these technologies. On the other side, one can also interpret the $f$ parameter as representing the perceived risk of losses of privacy (in which case the expected losses enter $f$ with a negative sign). In other words, the costs of privacy related decisions depend on two factors: short-term monetary costs or hassles of protective technologies or strategies, and the longerterm expected value that each customer forecasts she will obtain from using an anonymizing technology. This value may be expressed in negative terms when it represents the avoidance of the expected future random shocks due to the loss 
of privacy. If customers are not aware of the risks they incur when they do not protect their privacy, they will not consider the negative part of $f$, which means that even relatively cheap privacy technologies will not be adopted. Hence, they will choose not to protect their privacy (see Acquisti 2004, where privacy issues other than those associated with price discrimination are also discussed).

\subsection{Offering enhanced services}

In IT rich environments (and in particular in ubiquitous computing environments) the merchant can, however, offer something back to customers in exchange for using their information for dynamic pricing. For example, the merchant could make additional purchases by the same customer more efficient or pleasant through "enhanced services" of some form, enabled by the information the customer has revealed during the first purchase, and made possible by a system of sensor networks and mobile devices that channel the merchant information to the consumer and the consumer's decision to the merchant. Practical applications could be targeted recommendations, personalized service or content, one-click shopping, or a variety of other enhanced services discussed in Acquisti and Varian 2005. For example, in a grocery application, the system may identify the user and provide information through the shopping cart display about missing items on the shopping list Kourouthanassis and Roussos 2003a.

Acquisti and Varian Acquisti and Varian $\mid 2005$ study the scenario in which sellers can make the second unit of consumption more valuable than the first unit of consumption, and whether this can lead to increased profits. They find out that conditioning prices can maximize profits if the high-value purchaser values the enhanced services relatively more than the low-value user: for example, automated check-out through smart wireless wallets may be more valuable to consumers who shop more frequently or with higher values of time, which may be positively correlated with their valuation for the good itself.

The analysis holds for marginal costs of offering enhanced service that are lower than the customers' evaluations of those services. After the investment in information technology to track customer purchases and provide enhanced services is made, the marginal costs associated with each additional transactions are generally very low. One of the economic features of information technology is, often, the reduction of the marginal costs associated with certain repeated transactions and interactive marketing. Therefore it is reasonable to expect that after large initial investments to set up systems with enhanced services, the marginal costs of direct marketing and customer care for IT- and ubiquitous computing- focused sellers may be low. Large investments in IT might pay off in terms of the subsequent ability for the seller to offer particular services that competitors cannot provide, offering both a barrier to entry and benefits from price discrimination. In this respect, value-creating uses of ubiquitous computing technologies may be those associated with low marginal cost offers based on previous investments in information technology. 


\subsection{Making the second visit more valuable}

In Section 4.2 we referred to the case in which the second unit of consumption has a different value than the first. However, it may be that even if the consumer chooses not to purchase on the first visit to a seller, the second visit to the same merchant may generate higher value for the consumer. For example, registration, even without purchase, may lead to benefits such as automated checkout or personalized information. Certain stores, enhanced by ubiquitous computing technologies, may ask their visitors to create accounts before they can see products and prices and make any purchase. Hence the enhanced features considered in Section 4.2 might be offered to all returning customers whether or not they have purchased on their first visit. How does this affect the analysis?

We modify the model presented in Acquisti and Varian 2005 to answer this question. Let $v_{H 1}$ represent value of consumption for the high-value consumer during her first visit, and $v_{H 2}$ the value of consumption during her second visit. Define $v_{L 1}$ and $v_{L 2}$ similarly. The self-selection constraints now become

$$
\begin{aligned}
v_{H 1}+v_{H 2}-p_{H} & \geq v_{H 2}-p_{L} \\
v_{H 1}+v_{H 2}-p_{H} & \geq 0 \\
v_{L 2}-p_{L} & \geq v_{L 1}+v_{L 2}-p_{H} \\
v_{L 2}-p_{L} & \geq 0,
\end{aligned}
$$

The optimal prices are $p_{H}=v_{H 1}+v_{L 2}$ and $p_{L}=v_{L 2}$. The revenue from price conditioning exceeds the revenue from flat pricing when

$$
\begin{aligned}
& \pi v_{H 1}+v_{L 2}>\pi v_{H 1}+\pi v_{H 2} \\
& \pi v_{H 1}+v_{L 2}>v_{L 1}+v_{L 2} .
\end{aligned}
$$

Making the obvious cancellations gives the following result: conditioning prices will be optimal when

$$
\begin{aligned}
v_{L 2} & >\pi v_{H 2} \\
\pi v_{H 1} & >v_{L 1} .
\end{aligned}
$$

in which case $p_{H}=v_{H 1}+v_{L 2}$ and $p_{L}=v_{L 2}$.

Note that these inequalities are precisely the reverse of those summarized in Section 4.2 and described in Acquisti and Varian 2005. Comparing those results to those presented here, we can summarize what happens for all four sign patterns in these inequalities:

- $v_{L 2}>\pi v_{H 2}, \pi v_{H 1}>v_{L 1}$. Conditioning profitable when second visit has higher value.

- $v_{L 2}<\pi v_{H 2}, \pi v_{H 1}<v_{L 1}$. Conditioning profitable when second purchase has higher value.

- $v_{L 2}<\pi v_{H 2}, \pi v_{H 1}>v_{L 1}$. Sell only to high-value type. 
- $v_{L 2}>\pi v_{H 2}, \pi v_{H 1}<v_{L 1}$. Sell to both high- and low-value type.

In other words, a seller able to track consumers over repeated transactions and condition prices depending on previous purchase histories may have several different available strategies: flat-pricing to all consumers or just to the highvalue consumers, or adopt pricing strategies that induce conditioning. In the latter case, the seller may choose to provide enhanced services to all consumers visiting the seller's store a second time, or just to the consumers who already purchased on that store. Which strategy is the best will depend on the values of the parameters in this economy, and in particular on $\pi, v_{H 1}$, and $v_{L 1}$. Based on its perceptions of how many high and low value consumers exist in that economy, and how much each type of consumer is willing to pay for the good, the seller can choose its offerings so as to make the second purchase or visit a better experience, depending on which is more profitable. For example, when $\pi v_{H 1}<v_{L 1}$, the seller will find it optimal to make all consumers purchase in the first period and then offer the enhanced services to the returning high-value consumers in the second period.

\subsection{Making it costly (or not) to visit the seller}

This analysis, however, triggers a question: we have not explicitly considered the costs of visiting the seller. Should such costs be included in the price of the good purchased by the buyer, or in the cost of visiting the seller even if there are no purchases? In the model presented in Section 3, it would appear that the costs of inserting personal information (for example, stopping at the checkout counter to provide credit card data) the first time one purchases with a certain merchant could be incorporated in the model into the price that the customer is paying in the first period. Similarly, the enhanced service associated to the second purchase might be interpreted as the time saved the second time around (e.g., no-stop checkouts thanks to "intelligent" wireless wallets that interact automatically with the checkout counter).

Interestingly, it turns out that different types of tracking technologies may lead to slightly different solutions in the model.

A first type of tracking technology is a technology that requires user intervention (for example, manual registration of personal information on a wireless payment card at a ubiquitous computing equipped grocery store). A second type is a technology that works automatically and does not need user intervention (for example, automated registration of personal data through exchange of cryptographic keys and digital signatures between the user's devices and the store's sensors).

\subsubsection{Adopting a tracking technology that requires user intervention}

Let us consider first the technology that requires user intervention. Let us assume that the customer has to register every time she makes a purchase, because the seller is not storing any information. Then the profits for the seller 
from flat pricing will be either $2 v_{L}-2 d_{L}$ or $2 \pi v_{H}-2 \pi d_{H}$, where $d_{X}$ stands for the disutility of providing information to complete a purchase (for example, the subjective value assigned to the time spent doing that). Note that for this base case we assume that this information must be provided every time there is a purchase.

Compare this to the profits from price discrimination: the self-selection constraints produce the following optimal prices, $p_{L}=v_{L}-d_{L}$ and $p_{H}=$ $v_{H}+v_{L}-d_{L}-d_{H}$, and therefore the following profit: $\pi\left(v_{H}-d_{H}\right)+v_{L}-d_{L}$. By comparing these profits, one gets again the contradiction found in Section 3 conditioning, again, is not optimal.

Now consider what happens if the merchant stores the information so that the customer does not need to provide it again during the second purchase. Imagine that this is the only "enhanced service," that is, that the product itself is valued the same in both periods, and nothing more (no personalized offers, no targeted discounts) is being offered. The conditioning prices now will be: $p_{L}=v_{L}-d_{L}$ and $p_{H}=v_{H}+v_{L}-d_{L}$ (with a resulting profit: $\pi v_{H}+v_{L}-d_{L}$ ), while the profits from flat pricing will now be: $2 v_{L}-d_{L}$ or $2 \pi v_{H}-\pi d_{H}$. Conditioning will dominate flat pricing if

$$
\begin{aligned}
\pi v_{H} & \geq v_{L} \\
v_{L}+\pi d_{H}-d_{L} & \geq \pi v_{H} .
\end{aligned}
$$

These results are similar to the "second visit has different value" case discussed above. Inequalities 15 say that, even though the second purchase in this scenario has actually the same value, if the discomfort of the high type for spending time providing personal information is proportionally higher than the discomfort of the low type, then conditioning is optimal. This confirms that hassles (e.g. search costs) associated with making a purchase can be used to price discriminate among customers.

What happens when the "second visit has different value" in this setup? That is, what happens when the customer has to spend time logging in the first time regardless of whether she purchases or not the good? The above solutions do not change. In other words, when one approaches the price discrimination problem as a "cost of inserting personal information" problem rather than an "advantages from enhanced services" problem, it turns out that two cases are possible. In the first case, when the tracking technology requires time to be used (for example because the user has to register manually), then the "costs of inserting personal information" approach does not highlight any difference between one case and the other. In the second case, when the second visit has higher value because in the first visit the customer spent time registering, the self-selection constraints become equivalent to the case where the customer registered and purchased during the first period. 


\subsubsection{Adopting a tracking technology that does not require user intervention}

Things are different when one considers the second technology - a tracking technology which does not cost time or efforts to the customer even during her first visit or purchase but still offers her some advantages the second time she is around. This might happen for example because a vendor has automatically assigned a ubiquitous computing device to the customer (say, on a "smart" shopping cart) and through that device can track the customer's behavior. The vendor might observe which products the customer is passing by in the store or placing in the cart. The next time the same customer comes to that store, the seller may provide special offers on the customer's device, personalized and targeted based on what the customer's preferences have been revealed to be, without her having previously spent time explicitly compiling a profile or actually purchasing. In this case, indeed, price discrimination on the base of the "second visit having different value" becomes a more appealing strategy for the seller.

We can also combine the two approaches proposed in Section 4.2 and here. In the setup proposed in this section an implicit assumption was that the only benefit from a tracking technology is the saved time the second time around, equalled to (not having to waste) $d_{X}$. Now we can imagine that the customer values both the time saved and also additional enhanced services such as personalized information or targeted offers.

By mixing the two approaches in the self-selection constraints we get the following optimal prices in the conditioning case: $p_{L}=v_{L 2}-d_{L}$ and $p_{H}=$ $v_{H 1}+v_{L 2}-d_{L}$. The profits from flat pricing are: $\pi\left(v_{H 1}+v_{H 2}-d_{H}\right)$ and $v_{L 1}+v_{L 2}-d_{L}$. Conditioning will be optimal if

$$
\begin{aligned}
\pi v_{H 1} & \geq v_{L 1} \\
v_{L 2}+\pi d_{H}-d_{L} & \geq \pi v_{H 2}
\end{aligned}
$$

which is again a similar result to the one found above for the "second visit has different value" case. The results of this section therefore support the view that merchants can make strategic use of the heterogeneity in their customers' search costs (see also Zettelmeyer [1998]) for profitable price discrimination.

\subsection{Offering multiple goods}

So far we have analyzed price discrimination strategies that impose a separating equilibria: low type consumers buy once, and high type consumers buy twice a repeated purchase good. Consumer tracking data however can also be used for price discrimination without this form of rationing, when the seller is able to offer new but related goods at each visit. This strategy has the advantage for seller (but a disadvantage for the buyer) of making price discrimination less visible - because purchase histories are used to vary prices of different goods. 
This strategy is particularly relevant to ubiquitous computing commerce environments in which multiple related goods may be offered in the same physical or virtual space.

Imagine that in period 1 consumers can choose between two goods offered by a monopolist producer: $A_{1}$ and $A_{2}$. One type of consumer (let us call her the high type) prefers $A_{1}$ to $A_{2}: v_{H_{A_{1}}}>v_{H_{A_{2}}}$. The other type (the low type) prefers $A_{2}$ to $A_{1}: v_{L_{A_{2}}}>v_{L_{A_{1}}}$. Let us also assume that the seller knows that there are, again, $\pi$ high consumers, and that those high consumers are willing to pay a price $B_{H}$ for a third good $B$, while low consumers only value that good $B_{L}$, with $B_{H}>B_{L}$. For example, $A_{1}$ and $A_{2}$ could be, respectively, a CD with music by Gerswhin and a CD with music by Pink Floyd, and $B$ is a CD with music by Louis Armstrong.

What kind of pricing schemes the seller can adopt to elicit truthful revelation of each consumer's taste in period 1, in order to offer the third good at different prices in period 2? Again we adopt a mechanism design approach and try to solve for:

$$
\begin{aligned}
v_{H_{A_{1}}}+B_{H}-p_{H} & \geq v_{H_{A_{2}}}+B_{H}-p_{L} \\
v_{H_{A_{1}}}+B_{H}-p_{H} & \geq 0 \\
v_{L_{A_{2}}}+B_{L}-p_{L} & \geq v_{L_{A_{1}}}+B_{L}-p_{H} \\
v_{L_{A_{2}}}+B_{L}-p_{L} & \geq 0 .
\end{aligned}
$$

This system solves for $p_{L}=v_{L_{A_{2}}}+B_{L}$ and $p_{H}=v_{L_{A_{2}}}+B_{L}+\left(v_{H_{A_{1}}}-v_{H_{A_{2}}}\right)$. For example, the seller may charge $v_{L_{A_{2}}}$ for both goods in period one, and then either $B_{L}$ or $B_{L}+\left(v_{H_{A_{1}}}-v_{H_{A_{2}}}\right)$ for the second product in period two - depending on the customer's choice in period 1 .

Note that now both types are consuming twice. The high type, however, by revealing her preferences in period 1 , is charged an higher price than the low type in period 2 for the very same good. The profits are: $v_{L_{A_{2}}}+B_{L}+\pi\left(v_{H_{A_{1}}}-v_{H_{A_{2}}}\right)$.

Now, compare the conditioning profits to those obtained under

- flat pricing low $\left(\pi v_{H_{A_{1}}}+(1-\pi) v_{L_{A_{2}}}+B_{L}\right)$, and

- flat pricing high $\left(\pi v_{H_{A_{1}}}+(1-\pi) v_{L_{A_{2}}}+\pi B_{H}\right)$.

It turns out that conditioning is always better than flat pricing low, but is better than flat pricing high only when $\left(v_{L_{A_{2}}}-v_{H_{A_{2}}}\right) \geq\left(B_{H}-B_{L} / \pi\right)$ and the incentive compatible and individual rationality constraints above are satisfied (hence, for example, it must also be: $\left(B_{H}-B_{L}\right) \geq\left(v_{L_{A_{2}}}-v_{H_{A_{2}}}\right) \geq$ $\left.\left(B_{H}-B_{L} / \pi\right)\right)$. These inequalities draw upper and lower boundaries of the parameters under which price conditioning with repeated purchases by both types is optimal.

The conclusion is that tracking customer data can be used to profitably price discriminate even when the cost function is linear and no enhanced services are offered, if consumers have different evaluations for different goods offered across two periods. 
Note that under the proposed pricing strategy, when all constraints are met, no type has incentives to skip purchasing for a period, or using anonymizing technology. However, we have also implicitly assumed that the seller can commit to those prices.

One may notice that this form of price conditioning may be harder to detect for the consumer, since she is no longer dealing with a repeated purchase good. As the number of periods extends beyond two, it becomes increasingly difficult for any customer type to misrepresent herself for a different type, since this would imply suppressing at each period her real taste (or preferences).

Still, the power of information technology can also be used by consumers to monitor the seller's actions: in the famous Amazon.com price "experiment" (see Streifield 2001]), reports that the same product was being sold at different prices quickly spread from an Internet chat room to the mainstream media. Note, also, that in this chapter we have not considered additional privacy concerns associated with tracking technologies but not related to pricing: see Acquisti 2002 for a discussion.

\section{Implications}

Ubiquitous computing technologies offer retailers new opportunities to interact with their customers. Aggregating and analyzing purchase and individual data, sellers can provide personalized services, targeted offers, but also employ dynamic pricing strategies. In this chapter we have used micro economic theory to discuss under what conditions it may make sense for retailers to use ubiquitous computing tracking technologies to enforce price discrimination. Fleisch and Tellkamp 2003 point out that identifying value-creating uses of ubiquitous computing technologies is not trivial: we have shown here that naïve price discrimination may in fact be sub-optimal for sellers under general conditions.

We have shown under what conditions price discrimination based on tracking data may become optimal. First of all, the seller may provide enhanced services Acquisti and Varian 2005 that create the proper incentives for consumers to self-select into those who accept the higher prices as a cost to access desirable services, and those who prefer to receive lower prices - and lower quality services attached to the product they purchase. Therefore, sellers may deploy ubiquitous computing in ways that make the shopping experience for the consumer more engaging Kourouthanassis and Roussos 2003b or augment products with services. We have considered other strategies, such us making it difficult for customers to hide previous behavior through privacy enhancing technologies, or providing tracking and enhanced services which do or do not require customer intervention to operate. In this respect, a ubiquitous computing merchant may make strategic use of the heterogeneity in their customers' search costs through different forms of tracking technologies.

We have also considered the strategy of using customer's revealed preferences for one good as a tool for price discrimination on another good - a natural application in ubiquitous computing environments rich of different, related products, 
such as "smart" grocery stores.

With regard to this, Acquisti Acquisti 2003 shows under what conditions it is optimal for sellers to use previous purchase histories to recommend new goods to customers and gain from the customers' following the recommendations. The conditions studied in Acquisti 2003 may well apply to the ubiquitous computing commerce scenario, again for the case of merchants providing several goods and able to correlate data from several customers' transactions. Such recommendations may be useful particularly to sell niche goods for which customer's experimentation would otherwise be too costly.

Our results (and those in Acquisti and Varian 2005]) indicate that industries where transactions can be mediated through computers and in which the marginal costs of providing products augmented with personalized services are low (but those services show significant variation in user valuation) can be candidate for value-creating uses of ubiquitous computing technologies.

Of course, the complexity of certain ubiquitous computing environments may make it difficult for customers to recognize different pricing strategies offered by the seller. However, technology (for example, intelligent agents - see Sadeh et al. 2003]) may help consumers navigate through the complexities of the new pricing models made possible by ubiquitous tracking technologies.

\section{Acknowledgments}

I would like to thank Hal Varian, George Roussos, and the Information Technology department at HEC Montreal for help and discussions about the topics and models presented in this chapter. All errors remain my own. 


\section{References}

Alessandro Acquisti. Protecting privacy with economics: Economic incentives for preventive technologies in ubiquitous computing environments. In Workshop on Socially-informed Design of Privacy-enhancing Solutions, International Conference on Ubiquitous Computing (UBICOMP '02), 2002.

Alessandro Acquisti. Inducing customers to try new goods. In Workshop on Information Systems and Economics (WISE '03), 2003.

Alessandro Acquisti. Privacy in electronic commerce and the economics of immediate gratification. In Proceedings of the ACM Conference on Electronic Commerce (EC '04), pages 21-29, 2004.

Alessandro Acquisti and Hal R. Varian. Conditioning prices on purchase history. Marketing Science, forthcoming, 2005.

Robert C. Blattberg and John Deighton. Interactive marketing: Exploiting the age of addressability. Sloan Management Review, 33(1):5-14, 1991.

Giacomo Calzolari and Alessandro Pavan. Optimal design of privacy policies. Technical report, Gremaq, University of Toulouse, 2001.

Sylvia Carr. M-commerce market set to multiply. Silicon.com, October 282004. URL http://www. silicon.com.

Yuxin Chen and Ganesh Iyer. Consumer addressability and customized pricing. Marketing Science, 21(2):197-208, 2002.

Yuxin Chen, Chakravarthi Narasimhan, and Z. John Zhang. Individual marketing with imperfect targetability. Marketing Science, 20(1):23-41, 2001.

Oliver Christ, E. Fleisch, and Friedemann Mattern. M-Lab - The mobile and ubiquitous computing lab, Phase II. Technical report, M-Lab, 2003.

Elgar Fleisch. Business perspectives on ubiquitous computing. M-Lab Working Paper No. 4, University of St. Gallen, 2001.

Elgar Fleisch and Christian Tellkamp. The challenge of identifying valuecreating ubiquituous computing applications. In Workshop on Ubiquitous Commerce, International Conference on Ubiquitous Computing (UBICOMP '03), 2003.

Elgar Fleisch and Christian Tellkamp. The business value of ubiquitous computing technologies. In G. Roussos, editor, Ubiquitous Commerce. Springer Verlag, forthcoming, 2005.

Drew Fudenberg and Jean Tirole. Game Theory. MIT Press, Cambridge, MA, 1991. 
Anatole Gershman. Ubiquitous commerce: Always on, always aware, always pro-active. In Panel on Mobile Commerce: Vision and Challenges, International Symposium on Applications and the Internet (SAINT '02), 2002.

Oliver D. Hart and Jean Tirole. Contract renegotiation and coasian dynamics. Review of Economic Studies, 55(4):509-540, 1988.

Panos Kourouthanassis and George Roussos. Developing consumer-friendly pervasive retail systems. IEEE Pervasive Computing., 2(2):32-39, 2003a.

Panos Kourouthanassis and George Roussos. Developing the user experience in ubiquituous commerce. In Workshop on Ubiquitous Commerce, International Conference on Ubiquitous Computing (UBICOMP '03), 2003b.

Robert E. McCulloch, Peter E. Rossi, and Greg M. Allenby. The value of purchase history data in target marketing. Marketing Science, 15(4):321-340, 1996.

John Riley and Richard Zeckhauser. Optimal selling strategies: When to haggle, when to hold firm. Quarterly Journal of Economics, 98(2):267-289, 1983.

Peter E. Rossi and G. M. Allenby. Marketing models of consumer heterogeneity. Journal of Econometrics, 89(1-2):57-78, 1999.

George Roussos. Consumers and ubiquitous commerce. In Ubiconf 2004, April 19th, Gresham College, London, 2004.

Norman M. Sadeh, Ting-Chak Chan, Linh Van, OhByung Kwon, and Kazuaki Takizawa. Creating an open agent environment for context-aware mcommerce. In Agentcities: Challenges in Open Agent Environments, pages 152-158. Springer Verlag, 2003.

Stephen W. Salant. When is inducing self-selection suboptimal for a monopolist? Quarterly Journal of Economics, 104(2):391-397, 1989.

Nancy Stokey. Intertemporal price discrimination. Quarterly Journal of Economics, 93(3):355-371, 1979.

David Streifield. On the web price tags blur: What you pay could depend on who you are. The Washington Post, September 272001.

Curtis R. Taylor. Private demands and demands for privacy: Dynamic pricing and the market for customer information. Technical report, Duke University, Economics Department, 2002.

David Ulph and Nir Vulkan. Electronic commerce and competitive first-degree price discrimination. Technical report, University College, London, 2000.

David Ulph and Nir Vulkan. E-commerce, mass customization and price discrimination. Technical report, University College, London, 2001. 
J. Miguel Villas-Boas. Dynamic competition with customer recognition. RAND Journal of Economics, 30(4):604-631, 1999.

J. Miguel Villas-Boas. Price cycles in markets with customer recognition. RAND Journal of Economics, 35(3):forthcoming, 2004.

Mark Weiser. Some computer science issues in ubiquitous computing. Communications of the ACM, 36(7):75-84, 1993.

Florian Zettelmeyer. The strategic use of customer search cost. Technical report, University of California, Berkeley, 1998. 